\title{
Predicting disordered speech comprehensibility from Goodness of Pronunciation scores
}

\author{
Lionel Fontan $^{1}$, Thomas Pellegrini ${ }^{1}$, Julia Olcoz $^{2}$, Alberto Abad ${ }^{3,4}$ \\ ${ }^{1}$ Université de Toulouse; UPS; IRIT; Toulouse, France \\ ${ }^{2}$ ViVoLAB - Voice Input Voice Output Laboratory; I3A; Universidad de Zaragoza, Zaragoza, Spain \\ ${ }^{3}$ L2F - Spoken Language Systems Laboratory; INESC-ID; Lisbon, Portugal \\ ${ }^{4}$ IST - Intituto Superior Técnico, Universidade de Lisboa, Portugal \\ \{lionel.fontan, thomas.pellegrini\}eirit.fr, jolcozeunizar.es, alberto.abadel2f.inesc-id.pt
}

\begin{abstract}
Speech production assessment in disordered speech relies on tests such as intelligibility and/or comprehensibility tests. These tests are subjective and time-consuming for both the patients and the practitioners. In this paper, we report on the use of automatically-derived pronunciation scores to predict comprehensibility ratings, on a pilot development corpus comprised of 120 utterances recorded by 12 speakers with distinct pathologies. We found high correlation values $(0.81)$ between Goodness Of Pronunciation (GOP) scores and comprehensibility ratings. We compare the use of a baseline implementation of the GOP algorithm with a variant called forced-GOP, which showed better results. A linear regression model allowed to predict comprehensibility scores with a $20.9 \%$ relative error, compared to the reference scores given by two expert judges. A correlation value of 0.74 was obtained between both the manual and the predicted scores. Most of the prediction errors concern the speakers who have the most extreme ratings (the lowest or the largest values), showing that the predicted score range was globally more limited than the one of the manual scores due to the simplicity of the model.
\end{abstract}

Index Terms: pronunciation assessment, Goodness of Pronunciation, disordered speech, comprehensibility

\section{Introduction}

The assessment of speech production abilities in motor speech disorders relies almost exclusively on subjective tests such as intelligibility tests. These tests have two main disadvantages. They are very time-consuming and often imply subjective judgments: speakers read lists of words or sentences while one or several judge(s) evaluate their production. Within this framework automatic methods for speakers evaluation appear as practical alternatives. Recent advances in Automatic Speech Recognition (ASR) - especially in the field of Computer-Assisted Language Learning (CALL) - have contributed to develop techniques that may be of great interest for this purpose.

ASR techniques developed for the assessment of foreign language learners' pronunciation skills focused both on the segmental and the suprasegmental levels, giving birth to two research fields respectively called individual error detection and overall pronunciation assessment [1]. For individual error detection (i.e., automatic detection of mispronounced phones), two kinds of methods are used:

- methods based on the comparison of target phone models and learners' phone models (e.g. nonnativeness [2] or scores derived from classification methods such as linear discriminant analysis and alike [3]);

- methods independent of the learner's native language, such as raw recognition scores [4], or Goodness of Pronunciation scores (GOP [5, 6]).

Since the latter methods do not rely on any assumption concerning the errors possibly made by the speakers, their relevance may not be limited to the field of CALL. For example, GOP scores can be calculated to get an idea on how confident the ASR system is about each phone identity. In a previous research work [7], GOP scores were compared to perceptual analysis results in order to detect mispronounced phonemes in individuals with unilateral facial palsy (UFP). The algorithm was found to be effective: it detected $49.6 \%$ of mispronunciations (CR rate) and $84.6 \%$ of correct pronunciations. In [8] a preliminary test was conducted in order to study the relationship between mean GOP scores at sentence-level and subjective comprehensibility. Results were encouraging as highly significant correlations were observed, with absolute Pearson's coefficients ranging from .68 to .79 .

However, several questions remain concerning this last study. First, only the baseline implementation of the GOP algorithm was used. Recent algorithm refinements for CALL applications suggest that the accuracy of GOP results can be greatly improved, as in Forced-aligned GOP measurements (FGOP [9]). Moreover, the ability of GOP scores to predict comprehensibility judgments or measures was not assessed since the number of speakers was too limited. As a consequence the aim of the present work is twofold: 1) comparing the efficiency of GOP vs. F-GOP scores when dealing with disordered speech and 2) extending the number of speakers so as to test the ability of GOP measures to actually predict comprehensibility.

\section{GOP algorithms}

The purpose of the GOP algorithm is to automatically provide pronunciation scores at segmental level, that is one score per phone realization. The larger the score, the larger the difference between a phone realization and the corresponding phone model. In other words, large scores indicate potential mispronunciations. In this work, we used two different implementations: the original "baseline" one [5, 6], and a variant called Forced-aligned GOP (F-GOP) [9].

The baseline algorithm can be decomposed into three steps: 1) forced phone alignment phase, 2) free phone recognition phase and 3) score computation as the difference between the 
Table 1: Mean GOP values, reaction time and comprehensibility scores for 6 speakers. AP: Patients suffering from structural (anatomic) disorders, NP: Patients suffering from neurological disorders

\begin{tabular}{ccccc}
\hline Speaker & Mean GOP value & Mean F-GOP value & $\begin{array}{c}\text { Mean Reaction Time } \\
\text { to oral commands (s) }\end{array}$ & $\begin{array}{c}\text { Mean comprehensibility } \\
\text { score }\end{array}$ \\
\hline AP1 & $1.60(0.56)$ & $0.81(0.36)$ & $4.11(0.77)$ & $5.65(0.45)$ \\
NP1 & $2.32(0.66)$ & $1.11(0.38)$ & $4.63(1.08)$ & $5.30(0.40)$ \\
NP2 & $2.54(0.48)$ & $1.42(0.77)$ & $5.54(1.17)$ & $4.70(0.40)$ \\
AP2 & $2.86(0.71)$ & $1.99(0.58)$ & $5.50(1.20)$ & $4.05(0.45)$ \\
AP3 & $3.67(0.46)$ & $2.50(0.68)$ & $7.51(1.15)$ & $4.25(0.35)$ \\
AP4 & $4.15(0.67)$ & $4.01(1.18)$ & $9.64(2.56)$ & $1.65(0.25)$ \\
\hline
\end{tabular}

log-likelihoods of the two preceding phases for each forcedaligned phone. The forced alignment phase is intended to provide the ASR system with the orthographic transcription of the input sentence along with a pronunciation lexicon. It consists of forcing the system to align the speech signal with an expected phone sequence. On the contrary, free phone recognition determines the most likely phone sequence matching the audio input without constraint (free phone loop recognition). GOP scores typically range from zero (perfect match) to values up to 10 . Higher values often indicate that the aligning step failed for some reason and scores are meaningless in this case. In order to decide whether a phone was mispronounced ("rejected") or not ("accepted"), phone-dependent thresholds can be determined on a development set. In this work, our goal was not to detect individual mispronunciations but rather to compute average GOP scores per utterance in order to correlate them with comprehensibility scores given by human judges at utterancelevel.

The forced-aligned GOP version is exactly the same as the baseline one with the only difference that the phone boundaries found during forced alignment constrain the free phone recognition phase. For each aligned phone, a single phone is recognized. In [9], better correlations between GOP and manual scores were found with F-GOP than with baseline GOP in the context of a CALL experiment. Indeed, F-GOP removes the issues of comparing a single aligned phone with potentially several phones recognized within the same time interval.

\section{Main objective and methodology}

This study aims at verifying the ability of GOP measures to predict disordered speech comprehensibility. To this end, 12 pathological speakers were recorded. In a first experiment, these recordings were split in two subsets, each consisting of the sentences (imperative commands) recorded by 6 speakers: a development corpus and a test corpus (section 4). Reference comprehensibility scores, presented in section 5 , were obtained a) by asking 24 listeners to react to the sentences using software created for this purpose and $b$ ) by asking two trained speech pathologists to evaluate each sentence comprehensibility on a 7-points rating scale. Automatic measures found in GOP experiments (section 6) are compared so as to establish a predictive model of speakers' comprehensibility. This model is finally used to predict speech pathologists' comprehensibility judgments in 6 other patients (section 7). Since data from 6 speakers constitute a very small dataset with 60 utterances only, we also report prediction results in a cross-validation setup.

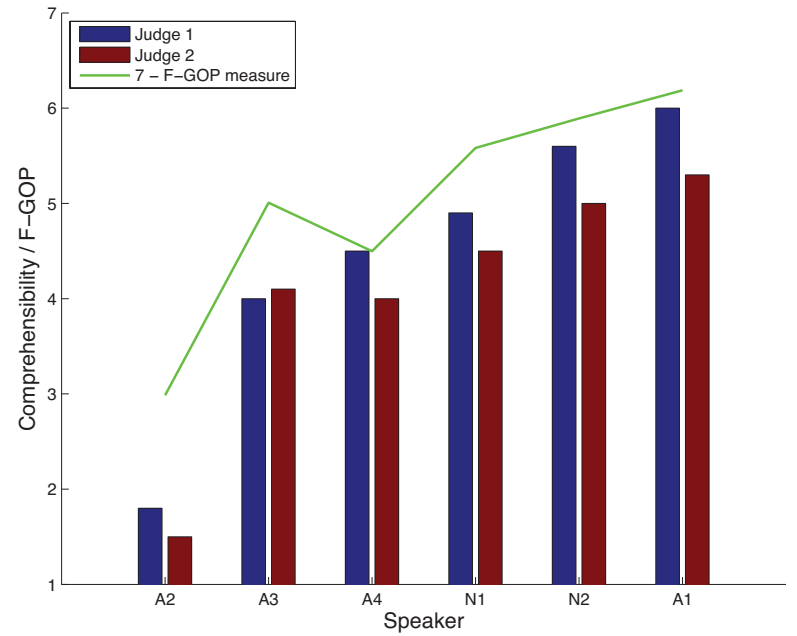

Figure 1: Comprehensibility judgments as a function of mean F-GOP scores. For a better clarity, F-GOP scores have been scaled following the equation: $y=7-F G O P$.

\section{Corpus description}

Speech stimuli were recorded from three female and nine male patients. Patients were aged from 33 to 70 years old (mean $=55$ ). Four patients suffered from speaking issues due to neurological disorders (spasmodic dysphonia, parkinsonian dysarthria (2) and Huntington's disease) and eight patients had troubles related to anatomic disorders: seven patients suffered from sequelae consecutive to oropharyngeal cancer surgery (among which two total laryngectomees) and one patient had dysphonia. The 12 patients were divided into two groups, both consisting in two patients suffering from neurological speech disorders and four patients suffering from anatomic speech disorders.

Each patient recorded 10 oral commands (sentences) among a hundred different ones, asking to move entities (animals or objects), such as "Mettez l'ours à gauche du kangourou" (Move the bear to the left of the kangaroo), or "Mettez le lion sous la banane" (Move the lion below the banana). All the commands had the same syntactic form. 

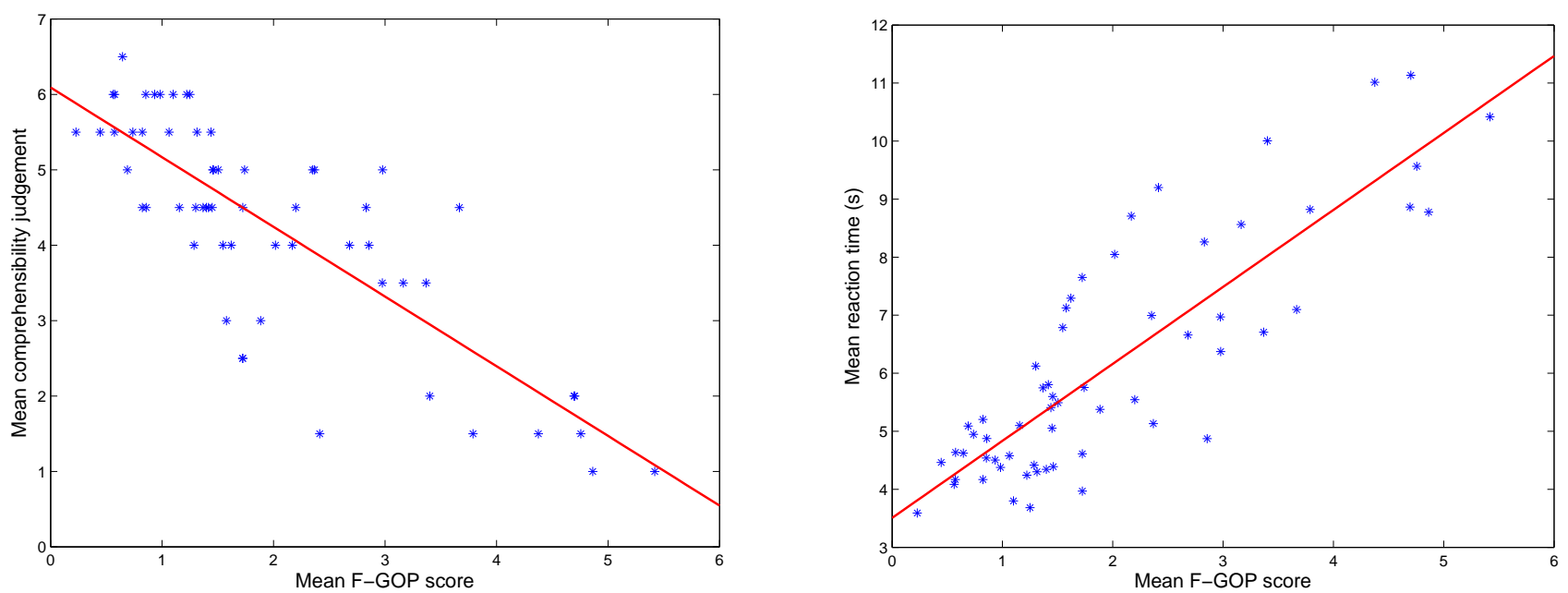

Figure 2: Left: Mean sentence comprehensibility as a function of F-GOP scores. Ratings range from 1 (very difficult to understand) to 7 (very easy to understand). The red line is regression fit of equation $y=-.92 * F-G O P+6.09$, Right: Mean reaction times to oral commands as a function of $F-G O P$. The red line represents the regression fit of equation $y=1.33 * F-G O P+3.51$,

\section{Comprehensibility measures}

\subsection{Subjective judgments of speech comprehensibility}

Two speech pathologists judged each sentence on a 7-points comprehensibility scale, ranging from 1 - very hard to understand up to 7 - very easy to understand. Both speech pathologists had more than 10 years of experience in listening and evaluating disordered speech. A Kendall tau-b rank correlation was computed so as to check the inter-rater agreement; a highly significant and strong correlation between the two rater scores was found $(t=.73 ; p<.001)$. Finally, mean subjective comprehensibility scores were calculated for each sentence by taking into account the two speech pathologists' grades.

\subsection{Behavioral scores: reaction times to oral commands}

Behavioral scores were collected for the 60 sentences forming the development corpus. For this purpose 24 listeners responded to the oral commands on a software created for recording their answers and reaction times [10]. For each command six images were displayed on a screen and listeners were asked to move the target image as demanded. As soon as the listener selected an image in order to move it, reaction time (RT) was collected. Keeping as an example the sentence asking to move the bear to the left of the kangaroo, RT was the time elapsed between the beginning of sentence play and the time at which the listener clicked on the image representing a bear. Only cases in which the listeners selected the right target image were considered. Listeners had a mean age of 32.5 years old $(S D=13.4)$ and benefited from various years of experience in listening to disordered speech $($ mean $=7.8 ; \mathrm{SD}=11.4)$. However, these two variables were found to have a comparable strength and opposite influence on RT [11]; consequently RT have not been weighted as a function of listeners' age and years of experience with disordered speech. Only mean RT for each sentence was taken into account.
Table 2: Pearson correlation coefficients between automatic scores and comprehensibility measures

\begin{tabular}{cc}
\hline Variables & Correlation \\
\hline GOP $*$ Comprehensibility ratings & $-.684^{* *}$ \\
F-GOP $*$ Comprehensibility ratings & $-.808^{* *}$ \\
GOP $*$ Reaction times & $.786^{* *}$ \\
F-GOP $*$ Reaction times & $.844^{* *}$ \\
\hline ** Correlation is significant at the .001 level (2-tailed)
\end{tabular}

** Correlation is significant at the .001 level (2-tailed)

\section{Relationship between GOP scores and speakers' comprehensibility}

This section is solely concerned with data issued from the development corpus. Results concerning the prediction of comprehensibility scores from the test corpus will be presented in section 7 .

\subsection{ASR system setup}

This work was carried out with HTK [12]. The acoustic models are three-state left-to-right HMMs with 32 Gaussian mixture components trained on the ESTER corpus [13]. As they have been found to be more suitable for CALL applications [14], context-independent acoustic models (39 monophones) were used.

\subsection{Results}

\subsubsection{Mean scores}

Table 1 presents mean and standard deviations of GOP and FGOP values as well as mean comprehensibility scores for each speaker of the development corpus. Mean RT tend to increase with mean GOP and F-GOP scores, whereas mean comprehensibility appears to decrease as a function of GOP and F-GOP. This suggests that the highest GOP and F-GOP scores are associated with the least comprehensible speakers, and vice versa. 


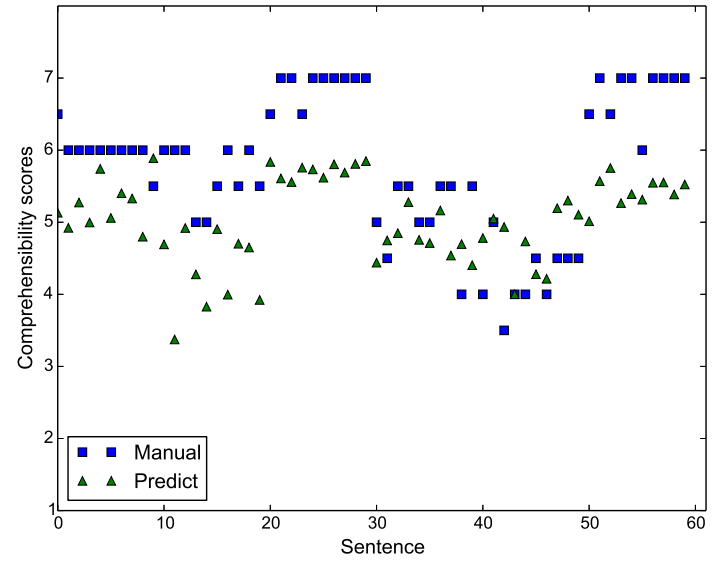

Figure 3: Manual and predicted comprehensibility scores for each sentence of the test group (6 speakers). Each speaker recorded 10 sentences, so sentences from 0 to 9 on the $X$-axis correspond to speaker A5, from 10 to 19 to speaker A6.

\subsubsection{Correlation between GOP scores and comprehensibility judgments}

Pearson product-moment correlation calculations were computed to study the relationship between GOP/F-GOP scores and comprehensibility measures. Results show a weaker correlation with GOP scores $(r=-.684 ; p<.001)$ than with F-GOP scores $(r=-.808 ; p<.001)$. Both correlations are negative, showing that comprehensibility judgments tend to increase as GOP scores decrease. To illustrate this, comprehensibility and mean F-GOP scores are represented in Figure 1. The correlation plot for all the sentences' F-GOP scores is shown on the left-hand side part of Figure 2.

\subsubsection{Correlation between GOP scores and reaction times}

For both GOP and F-GOP scores, Pearson product-moment correlation calculations indicate a strong and highly significant relationship with reaction times to oral commands. A stronger correlation is found with F-GOP scores $(r=.844 ; p<.001)$ than with GOP scores $(r=.786 ; p<.001)$. The correlation plot for F-GOP scores is shown on the right-hand side part of figure 2. All correlation coefficients found for GOP scores and F-GOP scores are presented in table 2.

\section{Prediction of speakers' comprehensibility}

As F-GOP are strongly correlated to the patients' comprehensibility scores, a second part of the present work focused on the ability of F-GOP scores to predict speakers' comprehensibility.

\subsection{Separate test set}

To this end, a first experiment consisted in estimating comprehensibility ratings for 6 "test" patients, different from the ones for which we reported results so far, with the help of the linear regression model previously described in Section 6.2.2. Predicted scores were compared to the mean comprehensibility rat-

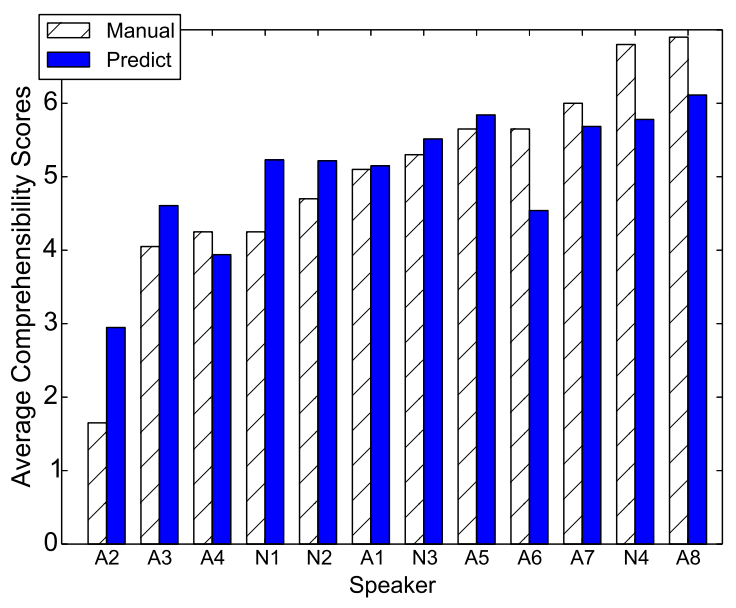

Figure 4: Mean manual and predicted scores for all the 12 speakers, obtained in the LOSO-CV setup.

ings given by the two speech pathologists. In figure 3 , manual and predicted comprehensibility scores are illustrated per sentence. Even if manual and predicted comprehensibility scores seem to follow the same tendencies $(r=.59)$ predicted scores appear to be globally lower than manual scores, with a $16.3 \%$ relative mean difference between both scores. This is mainly due to the fact that the 60 utterances were not sufficient to estimate a model.

\subsection{LOSO-CV setup}

In order to obtain sounder results, we repeated the experiment in a Leave-One-Speaker-Out Cross-Validation (LOSO-CV) fashion that allows to use more data to estimate the regression parameters. It corresponds to using data from 11 speakers (110 utterances) for the estimation of the regression parameters (slope and intercept), and to make predictions for the $12^{\text {th }}$ speaker that was left out. This process is repeated for each of the 12 speakers. A global Pearson correlation value of $r=.74$ was obtained, a much larger value than the preceding one. The relative mean difference is higher, though, with a value of $20.9 \%$. This is probably due to the fact that we make predictions for 12 speakers, twice as many speakers as in the preceding setup. Figure 4 shows a comparison of manual and predicted comprehensibility scores for all the 12 speakers. It shows that the dynamic range of the regression model is too limited: small and large scores are not predicted as accurately as medium scores.

\section{Conclusions}

The first noticeable result from this study is that a strong and highly significant relationship was found between GOP-derived scores and comprehensibility measures in the particular case of disordered speech. More precisely, the strongest correlations were found with F-GOP measures [9], which presented better results than conventional GOP scores [5]. This observation tends to present F-GOP scores as more closely related to speech production performance, as it was also observed in [9] and [14] in the application domain from which these two algorithms originate - namely Computer-Assisted Language Learning (CALL).

These encouraging results represented a strong motivation 
for studying the ability of F-GOP scores to predict disordered speech comprehensibility, which was done in the second part of this work. In a first score prediction experiment, data from 6 speakers (60 utterances) were used to estimate a simple linear regression model, and 60 comprehensibility automatic scores were predicted with this model on the remaining utterances from the 6 left-out speakers. A relative mean error of $16.3 \%$ was found, together with a low correlation value of 0.59 , when comparing the automatic and the manual scores. These results were not conclusive mainly because of the small size of the subset used to estimate the regression parameters. The same prediction experiment but in a cross-validation setup was more satisfying since a 0.79 correlation value was obtained. Nevertheless, the range of the automatic scores still was too small to correctly predict scores from speakers with low and large comprehensibility ratings.

As a response to these observations, future work will be devoted to the enlargement of the pathological speech data, by collecting speech representative of a wide variety of speech disorders. More complex regression models, such as Bayesian models, will be interesting to test. Such models allow to in troduce a priori information that may help in handling potential differences in model fits that may be seen for different groups of pathological speakers. Adding features characterizing suprasegmental aspects such as speech rate and pitch range, for instance, will also be worth testing.

\section{Acknowledgments}

The authors would like to acknowledge the Voice and Deglutition Unit (UVD) of the Hospital Larrey at Toulouse, with special regards to Virginie Woisard, Julie Bordenave and Astrid Ros, who participated in collecting the pathological speech data used in this study.

\section{References}

[1] M. Eskenazi, "An overview of spoken language technology for education,” Speech Communication, vol. 51, no. 10, pp. 832-844, 2009 .

[2] G. Kawai and K. Hirose, "A method for measuring the intelligibility and nonnativeness of phone quality in foreign language pronunciation training," in Proc. Internat. Conf. on Spoken Language Processing - ICSLP-1998, 1998, pp. 1823-1826.

[3] H. Strik, K. P. Truong, F. de Wet, and C. Cucchiarini, "Comparing classifiers for pronunciation error detection." in Proc. Interspeech 2007, 2007, pp. 1837-1840.

[4] B. Sevenster, G. d. Krom, and G. Bloothooft, "Evaluation and training of second-language learners' pronunciation using phoneme-based HMMs," in Proc. STiLL, Marholmen, 1998, pp. 91-94.

[5] S. Witt, "Use of Speech Recognition in Computer-Assisted Language Learning," PhD Thesis, University of Cambridge, Dept. of Engineering, 1999.

[6] S. Witt and S. Young, "Phone-level Pronunciation Scoring and Assessment for Interactive Language Learning," vol. 30, pp. 95$108,2000$.

[7] T. Pellegrini, L. Fontan, J. Mauclair, J. Farinas, and M. Robert, "The goodness of pronunciation algorithm applied to disordered speech," in Proc. Interspeech 2014, 2014, pp. 1463-1467.

[8] T. Pellegrini, L. Fontan, J. Mauclair, J. Farinas, C. Alazard-Guiu, M. Robert, and P. Gatignol, "Automatic assessment of speech capability loss in disordered speech," ACM Transactions on Accessible Computing, vol. 6:3, May 2015.
[9] D. Luo, Y. Qiao, N. Minematsu, Y. Yamauchi, and K. Hirose, "Analysis and Utilization of MLLR Speaker Adaptation Technique for Learners Pronunciation Evaluation," in Proc. Interspeech 2009, 2009, pp. 608-611.

[10] L. Fontan, P. Gaillard, and V. Woisard, "Comprendre et agir : les tests pragmatiques de comprhension de la parole et elokanz," in $\mathrm{La}$ voix et la parole perturbes, R. Sock, B. Vaxelaire, and C. Fauth, Eds. Mons: CIPA, 2013, pp. 131-144.

[11] L. Fontan, "De la mesure de l'intelligibilité à l'évaluation de la compréhension de la parole pathologique en situation de communication," PhD thesis, Université de Toulouse, 2012.

[12] S. Young and S. Young, "The HTK Hidden Markov Model Toolkit: Design and Philosophy," Entropic Cambridge Research Laboratory, Ltd, vol. 2, pp. 2-44, 1994.

[13] S. Galliano, E. Geoffrois, D. Mostefa, K. Choukri, J.-F. Bonastre, and G. Gravier, "The ESTER phase II evaluation campaign for the rich transcription of French broadcast news," in Proc. Interspeech 2005, 2005, pp. 1149-1152.

[14] T. Kawahara and N. Minematsu, Tutorial on CALL Systems at Interspeech, Portland, 2012. 